\title{
Visualisation Strategies in Abstract Animation
}

\author{
Ajay Kumar Tiwari \\ National Institute of Design \\ Paldi, Ahmedabad - 380007 \\ India \\ ajay_t@nid.edu
}

\begin{abstract}
The minimalist aesthetics and formalist tendencies of abstract animation correspond very well with the ease of experimentation with forms and movements and multiplicity of design options for image generation and manipulation offered by digital animation tools. In the paper the students' creative process of making abstract animation with digital and non-digital tools and materials is discussed within the context of abstraction in visual arts and experiments with forms and movements in the medium of animation.
\end{abstract}

Abstraction. Animation. Non-narrative. Minimalism. Synaesthesia.

\section{INTRODUCTION}

This paper discusses the visualisation techniques and strategies that the Postgraduate students have explored for artistic expression through abstract animation in the last five years during a course on Experimental Animation for New Media Design at National Institute of Design, India. The paper reflects on the students' creative process and their methods of visualizing forms and movements through intervention of digital tools in animation. It also presents a study on how the exploration of minimalist aesthetics in abstract animation through digital and non-digital methods broadens the creator's understanding of animation as an art form and offers an experience of the versatile language of animation beyond its narrative-communicative function.

\section{ANIMATION - A VERSATILE LANGUAGE}

Animation, as a medium of artistic expression, communication and experimentation, has always been elusive and has escaped all the attempts to define what animation is and what it can be. As a fundamental questioning and interrogation of the representational apparatus (Wells 2002a) and in its independence from any pre-existing reality to emerge purely in an animator's imagination, animation offers unprecedented creative possibilities in terms of innovative approaches towards mediums, materials and tools of creating forms and movements. This offers animation its uniqueness as a film form and presents it as an important facilitator of progress in the visual arts (Wells 2002a).
Versatility of the language of animation and its "undefinable-ness" offers the animators an open venue to experiment beyond conventional practices and explore variety of methods and tools to create animations that add new dimensions to an ever expanding vocabulary of animation.

Over the decades electronic and digital media have created avenues for new experiments with the visual language of animation and have helped animators, by offering them innovative ways of creative practices and artistic expression, to contribute in the evolution of animation as an art form.

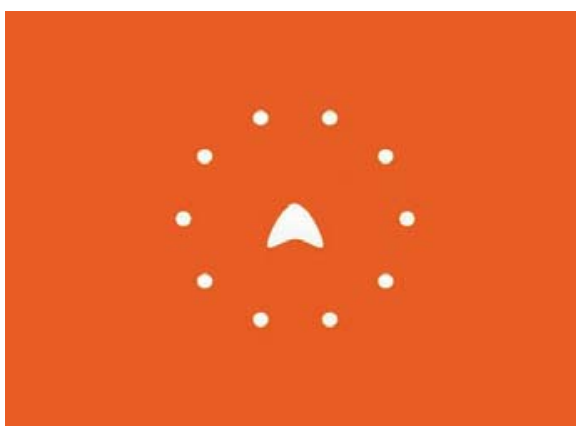

Figure 1: Film still from abstract animation "Dreams of Flight" by Nikhil Tailang (2012). Medium - Processing

\section{THE ART IN ABSTRACT ANIMATION}

Animation speaks to the material relationship between the artist and the means of expression and presents a widest possibility of materials, methods and tools to the animator to reveal or invent worlds (Wells 2002b). The animator has 
complete control over every single frame (the smallest visual unit in an animation film) in the process of translating ideas, observations and experiences into animated forms and movements. This presents animation as possibly the most "auteurist" of media (Wells 2002b).

"Complete authorial control" in all the stages of the creative process might have been the most significant and naturally appealing factor for the early artists, who were experimenting with abstract forms in motion, to explore the apparatus of animation as a platform to bring painting and cinema together (Gascard 1983) in an attempt to satisfy the kinetic urge that resulted from the extreme dynamism or quality of movement that the "overt-ambiguity" of abstract images had to offer (Gascard 1983). Many of the artists made abstract animations as inquiries into animation aesthetics (e.g., Norman McLaren) and formal investigations (e.g., Mary Ellen Bute and John Whitney) (Furniss 2009) where shapes, forms, colours and lines seem to act and react to take on a life of their own (Gascard 1983). The historian William Moritz suggested that inventing interesting forms and creating new imaginative, expressive motions in non-objective animation required the highest mental and spiritual faculties, as well as the most sensitive talents of hand (Wells 1998).

Abstract animation, even though it is still floundering for its aesthetic identity, forms a chapter of twentieth century art which refuses to be closed (Gascard 1983).

\section{ABSTRACTION IN VISUAL ARTS AND COMPUTERS AS CREATIVE COLLABORATORS}

Abstract animation, combining minimalist aesthetics with motion, can be seen as the most obvious playground available to the early "technological artists" as well as the painters. Painting and cinema have tended to cross paths, theoretically augmenting each other's development (Gascard 1983), as a response to the visual art's tendency towards formalism as well as the "kinetic urge" in the age of accelerated movement (Gascard 1983). Introduction of "motion" through the technical means of cinema in the early 20th century ended the long wait of painters and sculptors who had long attempted to suggest movement in their static works (Collopy 2000). Abstraction and Movement continued to be the important areas of visual experiments by artists, engaging into scientific inquiries as well, who explored computers as accelerators of "high-speed visual thinking" (Dietrich 1986) in the 1960s. According to Robert Mullary the computer worked as a tool for enhancing the creative power and productivity of the artist by accelerating the creative process and by making available multitude of design options that otherwise might not occur to the artist (Dietrich 1986). This in turn opened up many ways for experiments and investigations of forms and motions in visual arts.

Most of the early application of computers in visual arts explorations avoided reference to any content and were experiments to create visually exciting structures and explore the relationship between forms (Victoria \& Albert Museum 2014) where the artists, having no interest in elaborate painting, allowed themselves to relate to the simple imagery generated by computers (Dietrich 1986).

The computers, and the digital tools for artistic creation that were made available afterwards, provided computational power to the creative vision of the artists in generating abstract forms and movements and opened newer windows of visual experimentation which in turn led to development of a new vocabulary in the language of animation.

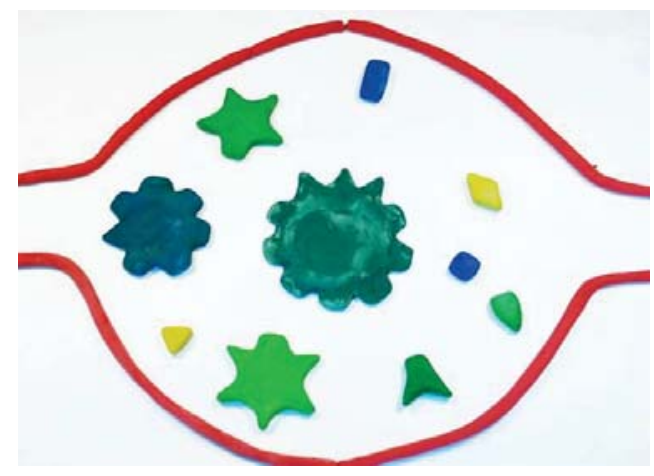

Figure 2: Film still from abstract animation "Dash" by Anuradha Reddy (2009). Medium - Clay on Glass

\section{MUSIC AND ABSTRACTION IN VISUAL ARTS}

Oskar Wilde suggested that music, an art in which form and matter are always one and where the subject and method of its expression are inseparable, truly realizes the artistic ideal and is the condition to which all other arts are constantly aspiring (Collopy 2000). Music, as pure abstraction and being absolutely self-reliant on its own form to communicate, has always been a source of inspiration for visual artists exploring abstraction and attempting to achieve a sense of musicality and music's formal self-reliance in their works. The Canadian animator Norman McLaren's intellectual inquiries in animation aesthetics and experiments in "pure design" (Mollaghan 2011) reflected minimalist tendencies that corresponded to minimalist aesthetics in music. Abstraction, as a common element in music and paintings, and Temporality, as a common element in music and cinema, find a perfect link in abstract animation 
where pure formal study and investigation takes over as primary objective of the artist. In abstract animation painting broke away from "immobility" which was considered as painting's last and principal shackle by the Cubist Painter Leopold Survage (Collopy 2000).

The artistic experiments exploring the association of visuals and music go a long way back. Arguably the first efforts to use technology to apply concepts of musical composition to abstract arts of motion came with a series of experimental devices from the 18th through the 20th centuries generally called "colour organs" (Alves 2005). In the first decades of 20th century artists like Scriabin, Kandinsky and Klee were interested in combining music and visual arts (Abbado 1988).

Historians of Abstract Animation also see its origins in attempts to link "and music and it may have had its roots in the psychological condition of synaesthesia (Lambert 2009). As compared to colour organs the technology of filmed animation offered much greater control over form and direct synchronisation to music (Alves 2005). Some of the most remarkable films exploring relationship of abstract visual forms and music were creation of early abstract animators like Oskar Fischinger and as gradually the computers offered artistic possibilities as image generators a whole new gamut of experiments in music and visual arts in motion emerged, e.g., in the computer animations of John Whitney. This added an altogether new dimension in the language of animation.

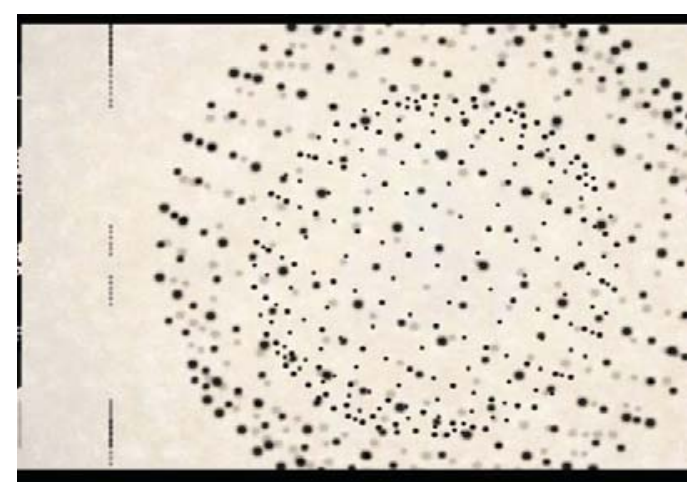

Figure 3: Film still from abstract animation "Realms: Experience and Change" by Abhinav Mishra (2011). Medium - Adobe Flash

\section{OBSERVATIONS ON THE STUDENTS' CREATIVE PROCESS}

\subsection{The constraints and the guidelines}

All the students were to use the music track designed by one of the minimalist composers, e.g., Philip Glass, Terry Riley, Steve Reich, La Monte Young and then visualize the music track using only basic shapes like points, lines, circles etc. What concepts or feelings were to be communicated or expressed were completely left to the students to arrive at in the creative process.

The journey of cinema (films and animation) and its evolution as an art form was the primary context of the course. The course comprised of lectures, screenings and hands-on exercises that aimed to introduce the students to the history of experimental films and animation. At the same time lecture sessions also invited critical discussions on social-political happenings, artists' works and philosophies, avant-garde tendencies and experimental practices in visual arts and music in the 20th century.

\subsection{Observations}

Following the students closely in their creative process I came across some observations with reference to their perception, practices and learning.

\subsubsection{Narrative + Representational versus Non- narrative + Abstract}

This course offered new perspectives and understanding about cinema. From being a medium of storytelling and pure entertainment to a medium of creative expression, artistic practice and philosophical investigation of the world and mind, one could see a drastic change in the students' perception about films and animation.

Abstract films and animation are "open texts" leading beyond the film itself and are often 'about' the need to expand our ability to see, experience, and comprehend things in day-to-day life. For that reason they challenge the viewer to participate in the process of creating meaning. Rather than following the story or assessing the interactions of representational figures, a viewer of abstract work typically must assume a much more intuitive and contemplative approach to the material (Furniss 2009).

The students took some time to settle for a nonnarrative approach in their process of exploring expressive and communicative potential of nonrepresentational, abstract elements, i.e., simple geometric forms. It was a slow but a gradual process for them to develop critical-reflective approach towards the medium as well as their own creative process while engaging in "formal" experiments and investigations.

Most of the students found abstraction challenging and intriguing at the same time and felt that abstraction's ambiguity and open-ended-ness open up more channels of understanding and interpretation while offering opportunities for a 
playful and active engagement with the works of art and thus provide a far more enriching experience.

\subsubsection{Concepts versus Emotions}

Many of my students experienced and explored abstraction in animation as a journey from the 'exterior' to the 'interior'. As their visual exploration in abstraction took them away from externalobjective reality into more of a reflective and contemplative mode, the students ventured into the avenues of mental, emotional (predominantly), psychological and spiritual and explored dreams, imaginations, phobias and hallucinations as potential sources of concepts. At the same time the constraints of abstraction kept the students away from "expressionistic" approaches that rely on manipulation and distortion of traditional representational means. Instead it let the students explore the realm of transcendental emotions (Rosenthal 1996) in their experiments with forms.

The students' relation to abstraction was primarily "emotional" which I realized was due to the presence of music as one of the constraints in the exercise (along with abstraction). Most of the students related to and responded to the music "emotionally", which I found quite natural as music has the power to reveal something about human life and experience and is a powerful vehicle for expression of emotions (Graham 1997). Although music "in itself" was a source of emotions and feelings that the students took forward in their visual exploration, the structure and dynamic of music was also of great significance when it came to conception of Forms and, at later stages, their transformation and movements over time. Thus music was not only a source of emotions but also provided structural complexities and possibilities to be analyzed and understood by the students in their formal visual exploration during animation.

Most of the students began exploring with the "emotional" content offered by the music and then moved on to abstract forms and their transformations and movements, following the dynamics offered by the music, in their process of animation. Synchronisation of Visuals and Sounds was the stage where "concept" took prominence to guide, along with music, the creative decisions in animation where experimentation with abstract visual forms in motion was the primary objective.

Some of the students on the other hand consciously moved away from concepts and emotions and concentrated solely on geometric forms to experiment with formal structures and relationships while keeping "synchronisation" as the central objective in their animation process. Here the "structural complexity and dynamism" of music were perfectly matched with "possibilities of transformations and movements in forms" and led to pure abstraction with complete objectivity while gradually moving into the realm of synaesthesia.

\subsubsection{Synchronisation versus Formal Investigation: Musical-Visual Dynamic}

There were two creative approaches among the students that I could observe. On one hand the starting point was either a concept or a feeling (mostly from students' experiences and observations or inspired by the music) which was taken forward into animation. On the other hand the students, who did not begin with an idea or an emotion, kept the structure of the music as the starting point and attempted to achieve the "musicality" in their visual-motion exploration as objectively as possible without referring to any content. "Complete objectivity" and "pure abstraction" happened to almost all the students at some point in their creative process where they stopped referring to the dynamics of the music, got away from all the concepts and feelings, stopped synchronisation and solely concentrated on transformation and movement possibilities offered by the forms. That approach could very well be called as "form for form's sake".

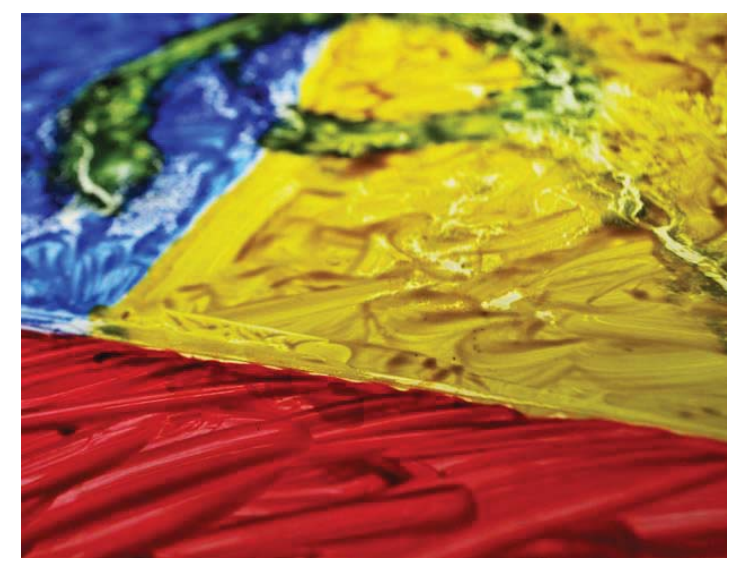

Figure 4: Film still from abstract animation "Mélange" by Annapurna Banerjee (2009). Medium - Oil Paint on Glass

In both the approaches the common factor was the constraints offered by the "minimalism" of abstraction. As simple abstract forms were all they had, to communicate and express through in their animations, the process helped the students expand the boundaries of their perception and understanding while exploring the possibilities and the potential of the abstract forms to the fullest.

Music did affect the animation of forms but mostly the origin of the forms was guided by visual abstraction of real life scenarios that the students portrayed in their creations. It was the concept that led to choosing or creating a specific Form while the dynamic in animation, in terms of forms' transformations and movements, was primarily governed by the music. 


\subsubsection{Analogue versus Digital - a dialogue}

As the students restricted themselves to only a few simple visual elements, e.g., dots, lines, circles, etc. digital tools offered them a venue to play around with forms, colours and movements while offering them a multitude of design options to choose from. The students found the digital tools to be less restrictive and imposing and more open to trial and error for exploring a greater number of creative possibilities in simple geometric forms.

When it came to programming for animation (Processing for example) versus choosing an animation software (Flash, AfterEffects), ease of use was a determining factor for many of the students as they realized that their animation was to a great extent about complex movements and transformations of simple forms which required control and manipulation of many properties (e.g., colour, shape, size, position, etc.) of the forms to achieve desired output. The digital tools also led the students push their forms conceptually further from what they had originally imagined and brought interesting surprises in terms of letting the students create variations in the forms that students themselves had not envisaged.

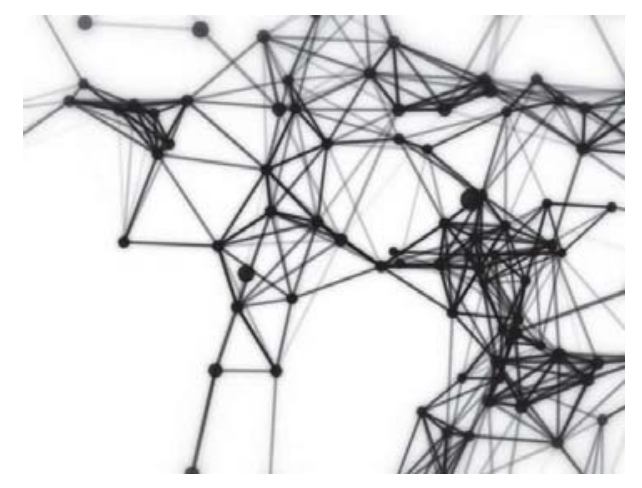

Figure 5: Film still from abstract animation "Labyrinth" by Sameer Ikararahmed Ansari (2013). Medium - Adobe AfterEffects

The students felt that their animation was certainly affected to some degree by the tool(s) they explored for making their animations. For example many of them felt that since computers can quickly create lots of variations and combinations based on the properties of elements that are being animated, it helps one start with what one has in mind and then see new developments in forms and movements as one goes on exploring. At the same time digital tools also provided the students a window to look at different attributes and properties of the chosen form as one keeps working on them, in close collaboration with the tool one is using, by tweaking properties of the forms and see if it looks interesting enough to be taken forward.

On the other hand limitations of the digital tools added another layer of constraints and guided the final animation output, just like the other constraints, i.e., abstraction and music. What is unique about the language of animation is its versatility in terms of variety of mediums, tools and materials that can be explored for creating animation and the choice of tools or materials decides to what extent the animator is going to not only explore the existing vocabulary of animation but also add something new to it. The tools and materials play a significant role in shaping the animator's thoughts and emotions into animated forms and affect the audience's experience of the world that the animator invents.

Different mediums (analogue and digital) allowed the students to explore different aspects of the language of animation with varying degrees of ease and efficiency. For example one gets much better creative control in morphing shapes in some mediums like hand drawn animation or clay animation as compared to exploring it in a digital medium like Flash or AfterEffects. But at the same time, generating variations in properties of forms is far more easy and effective in digital tools compared to creating them by hand. As animation, by its very definition, is all about inventing worlds and entities and bringing them to life through movements with an animator's creative spirit, the relationship between the artist and material is even more significant in case of animation as nothing is reproduced from real life (unlike live action films) but is brought to existence solely through an animator's creative play with the materials of different kinds (analogue and digital, i.e., clay, paper, pencil, codes or pure data).

In my observation it was quite evident that digital tools for animation provided a highly collaborative (between the animators and the tools) and creative environments for the students in their animation process. As the students restricted themselves to animating simple abstract forms in the beginning the tools played a significant part in guiding their visual language by offering instant feedback to the students' initial explorations of forms. The digital tools not only made the whole creative process faster but also provided multiplicity of transformation and movement options which were either different than what the students expected or were simply what the students did not even have in mind. Thus the students' abstract animation adventure was pure "formal" investigation and experimentation and was a highly enriching experience throughout.

\section{CONCLUSION}

Exploring abstract animation offered an avenue to the students to appreciate and practice the medium of animation as a tool for artistic expression and 
creative experimentation beyond its narrativecommunicative function. It also proved to be an exercise to experience the versatility of the language of animation at work where students engaged with a variety of materials, tools and methods from hand drawn to clay, oil on glass, cutout and a combination of digital tools including Flash, AfterEffects, Processing etc. But all the means were aiming towards the same end, i.e., experimenting with abstract visual forms in motion.

In their creative process the students followed different approaches when it came to selection of forms, arriving at the concepts or emotions to be translated into animation and deciding upon ways of exploring correspondence between the music and visuals. But one could clearly see a dialogue between the students' creative intent and the limitations and possibilities offered by the digital tools in terms of providing design options for generating, modifying and animating abstract forms. Although the core idea of animation, i.e., bringing inanimate entities to life through movements, remains the same in case of both analogue and digital mediums, the creative act of imparting motion to abstract forms and the digital animation tools seemed to go hand-in-hand where minimalism in abstraction fit quite well with the multiplicity of design options, for creation and animation of forms, offered by the digital tools. The tools worked as a utility, as well as creative collaborators in the students' design process, where the primary objective was to explore the language of animation for experimentation and investigation of visual forms in motion.

Arriving at a harmony between ambiguity and multiplicity of interpretation in abstraction, while exploring the creative possibilities in the dialogue between the artistic intention and the medium of animation, was a challenging and enriching experience at the same time. Animating nonrepresentational forms for creative expression, communication or simply experimentation added new dimensions to the students' perspective about the world and life as well as the art of animation.

\section{ACKNOWLEDGEMENT}

Dr Jignesh Khakhar, Dr Rita Kothari and Mohankrishnan Haridasan (co-faculties in the course). All the students of Post Graduate Diploma Program in New Media Design - from year 2009 to 2013.

\section{REFERENCES}

Abbado, A. (1988) Perceptual correspondences of abstract animation and synthetic sound. Leonardo, Electronic Art Supplemental Issue, pp. 3-5.

Alves, B. (2005) Digital harmony of sound and light. Computer Music Journal, vol. 29, no. 4, pp. 45-54.

Collopy, F. (2000) Color, form and motion: dimensions of a musical art of light. Leonardo, vol. 33, no. 5, pp. 355-360.

Dietrich, F. (1986) Visual intelligence: the first decade of computer art (1965-1975). Leonardo, vol. 19, no. 2, pp. 159-169.

Furniss, M. (2009) Considering form in abstract animation. In Art in Motion: Animation Aesthetics. John Libbey Publishing, UK.

Gascard, L. D. (1983) Motion Painting: 'Abstract' animation as an art form. Leonardo, vol. 16, no. 4, pp. 293-297.

Graham, G. (1997) Music and meaning. In Philosophy of The Arts: An Introduction to Aesthetics, Routledge, London, UK.

Lambert, N. (2009) A critical examination of 'computer art'. Computer Art Thesis. Archived 20/2/2012 by the Computer Arts Society. http://computer-arts-

society.com/static/cas/computerartsthesis/index.ht ml\%3Fpage id=111.html (accessed 18 May 2014).

Mollaghan, A. (2011) An experiment in pure design: the minimalist aesthetics in the line films of Norman McLaren. Animation Studies, vol. 6.

Rosenthal, M. (1996) The pioneers. In Abstraction in the Twentieth Century: Total Risk, freedom, Discipline. Solomon R. Guggenheim Foundation, New York.

Victoria \& Albert Museum. A History of Computer Art. http://www.vam.ac.uk/content/articles/a/ computer-art-history/ (accessed 14 May 2014).

Wells, P. (1998) Theory of animation. In Understanding Animation. Routledge, London, UK.

Wells, P. (2002a) Introduction: Abdicating all mental law. In Animation and America. Edinburgh University Press, Edinburgh.

Wells, P. (2002b) 'Animation is the most important art form of twentieth century' discuss. In Kearton, N. (ed.), Art \& Animation (Art \& Design), John Wiley $\&$ Sons, Ltd. 